\title{
Microdureza de resina composta fotopolimerizável: a cor da matriz experimental pode alterar os resultados dos testes?
}

\section{Microhardness of photopolymerized composite resin: can the color of the experimental matrix change the results of the tests?}

\author{
Míriam Lacalle TURBINO* \\ Luciana Aily SANTOS** \\ Edmir MATSON***
}

\begin{abstract}
TURBINO, M. L.; SANTOS, L. A.; MATSON, E. Microdureza de resina composta fotopolimerizável: a cor da matriz experimental pode alterar os resultados dos testes? Pesqui Odontol Bras, v. 14, n. 3, p. 232-236, jul./set. 2000.

O grau de polimerização das resinas compostas, avaliado principalmente pela microdureza vem sendo estudado relacionado com inúmeros fatores. Algumas pesquisas in vitro fazem uso de matrizes como base para a confecção de corpos-de-prova e não dentes naturais. Para as resinas fotopolimerizáveis, a intensidade de luz que atinge o material exerce influência direta no seu grau de polimerização. A cor e/ou transparência dessas matrizes não deveriam induzir a diferenças nos resultados finais de microdureza. Com essa preocupação, este trabalho teve por objetivo avaliar o efeito de duas matrizes de polipropileno utilizadas para testes com resina composta. Uma delas era branca e outra preta. A resina foi inserida e polimerizada em incremento único de $3 \mathrm{~mm}$ e em 3 incrementos de $1 \mathrm{~mm}$ cada e sua dureza medida a $3 \mathrm{~mm}$ de profundidade. Analisando os resultados pode-se concluir que houve diferença estatisticamente significante entre as duas cores, sendo que a microdureza foi menor com a matriz preta. A técnica de inserção e polimerização em incremento único induziu a menor grau de polimerização que a técnica incremental em ambas as matrizes $(\mathrm{p}<0,01)$. Pôde-se obter com esses resultados, que ao utilizar matrizes pretas para confecção de corpos-de-prova de resina composta fotopolimerizável, os valores obtidos, principalmente relacionados com profundidade de polimerização, demonstram o menor grau possivel de polimerização, e que depende exclusivamente das propriedades da resina.
\end{abstract}

UNITERMOS: Resinas compostas.

\section{INTRODUÇÃO}

Atualmente, em virtude da grande informação que chega ao nosso paciente através de propagandas de produtos odontológicos na TV, revistas de moda/beleza sobre cosmética e também via Internet, nos vemos cada vez mais na obrigação de estudar e melhorar as qualidades dos materiais estéticos.

Dentre eles, as resinas compostas para restaurações diretas são as mais utilizadas devido às suas características estéticas e facilidade de uso. Além disso, seu custo e tempo de confecção são menores quando comparado às restaurações indiretas.

As restaurações de resina composta, quando bem indicadas e bem executadas, aliadas aos cuidados de manutenção periódica pelo paciente, tornam-se trabalhos de excelente qualidade e estética funcionais ${ }^{14}$. Sabe-se que, embora esse tipo de res- tauração seja executado diariamente pelo cirurgião-dentista e com aparente facilidade, é um trabalho que exige cuidados durante sua manipulação no decorrer dos procedimentos restauradores. Ainda existem deficiências nas restaurações diretas de resina composta, como: contração de polimerização, inserção de bolhas fragilizando o dente, diferentes graus de polimerização conforme as espessuras, desgastes oclusais. Uma das propriedades avaliadas desse material é a dureza que tem sido analisada em testes in vitro com corpos-de-prova confeccionados em cavidades de dentes naturais ou em matrizes. Como matriz, tem-se utilizado molde de teflon ${ }^{7,8,15,17}$, matriz de teflon branco ${ }^{14}$ ou de poliacetato de coloração cla$\mathrm{ra}^{2,12,16,18}$, resina acrílica quimicamente $\operatorname{ativada}^{1} \mathrm{e}$ matriz plástica ${ }^{3}$. Também têm sido utilizadas matrizes de metal $^{10}$, molde de alumínio ${ }^{13}$, matrizes de aço $^{11}$, matriz de propileno polimetacrilato ${ }^{9}$.

* Professora Doutora; ** Pós-Graduanda em Nível de Mestrado; *** Professor Titular - Departamento de Dentística da Faculdade de Odontologia da USP. 
TURBINO, M. L.; SANTOS, L. A.; MATSON, E. Microdureza de resina composta fotopolimerizável: a cor da matriz experimental pode alterar os resultados dos testes? Pesqui Odontol Bras, v. 14, n. 3, p. 232-236, jul./set. 2000.

Além do material com que são confeccionadas, as matrizes também têm características diferentes, como formatos e tamanhos, os quais são descritos a seguir.

As matrizes utilizadas por DREXLER et $a l^{7}$ (1997), eram teflon com cavidade cilindrica de $3 \mathrm{~mm}$ de profundidade e diâmetro de $6 \mathrm{~mm}$ e o fator de variação era a utilização de cone plástico transparente sobre a resina, que segundo esse estudo aumentava a dureza de superficie da resina composta. A matriz de teflon utilizada por GALAN Jr. et al. ${ }^{8}$ (1984) e por CORADAZZI et al. ${ }^{4}$ (1986), tinha $12 \mathrm{~mm}$ de profundidade e $6 \mathrm{~mm}$ de diâmetro. DE GEE et al. ${ }^{6}(1981)$ e SANTOS et al. ${ }^{16}$ (1999) concordam que a inserção do material deve ser feita por incrementos a fim de obter maior dureza da camada mais interna e profunda do material, utilizando matriz de poliacetato de cor clara. PEREIRA et al. ${ }^{12}$ (1997) para avaliar intensidade de luz e profundidade de polimerização utilizaram três aparelhos fotopolimerizadores com a mesma matriz proposta por VINHA et al. ${ }^{19}$ (1990), cujo material era de poliacetato bipartido com uma cavidade de $7 \mathrm{~mm}$ de comprimento, por $4 \mathrm{~mm}$ de largura e $3 \mathrm{~mm}$ de profundidade.

ATMADJA; BRYANT ${ }^{2}$ (1990), mostraram que o grau de polimerização das resinas compostas ia diminuindo proporcionalmente ao aumento de sua profundidade. Para tal experimento, utilizaram molde cilindrico branco com $4 \mathrm{~mm}$ de diâmetro e 1 , 2, 3, 4 e $6 \mathrm{~mm}$ de espessura.

ANDRADE et al. ${ }^{1}$ (1996), estudando o efeito da aplicação de flúor sobre a dureza superficial dos cimentos de ionômero de vidro, confeccionaram matriz circular com RAAQ, com $1 \mathrm{~cm}$ de diâmetro, $1 \mathrm{~cm}$ de altura e o centro continha uma cavidade circular de $2 \mathrm{~mm}$ de profundidade e $5 \mathrm{~mm}$ de diâmetro.

Alguns autores utilizaram matrizes de materiais que não permitiam a passagem de luz, ficando restrita apenas à abertura delas. CORRER SOBRINHO $^{5}$ et al. (1995), prepararam um molde de latão para fazer amostras de $5 \mathrm{~mm}$ de diâmetro de espessura para avaliar a correlação de intensidade de luz emitida por aparelho fotopolimerizador e o tempo de exposição da luz ativadora sobre o grau de polimerização de um compósito odontológico ativado por luz visivel. Os resultados indicaram que os aparelhos com maior intensidade de luz proporcionaram maiores valores de dureza nas regiões de superfície e de fundo. A região de superficie apresentou valores superiores em relação ao fundo nos aparelhos com 130, 220 e $280 \mathrm{~mW} / \mathrm{cm}^{2}$, após 30, 45 e 60 segundos de exposição.
MARAIS et al. ${ }^{10}(1987)$ com uma matriz de metal com cavidade de $5 \mathrm{~mm}$ de diâmetro, colocavam a resina composta a fim de estudar sua dureza em profundidades diferentes em diferentes intensidades de luz. Já PILO et al. ${ }^{13}$ (1994), usaram moldes de alumínio para estudar o efeito do tempo de radiação na resistência de cisalhamento das resinas compostas.

TURBINO $^{18}$ (1997) utilizou matriz de propileno preta, avaliando a microdureza de resinas compostas inseridas em três incrementos ou incremento único na mesma profundidade de $2,6 \mathrm{~mm} \mathrm{e}$ verificou grande influência da espessura do incremento na dureza da resina composta.

Para as resinas fotopolimerizáveis, a intensidade de luz que atinge o material exerce influência direta no seu grau de polimerização. O material pelo qual é composta essa matriz, deve ser inerte às suas propriedades. Por conseqüência, sua cor e/ou transparência não deveriam induzir a diferenças nos resultados finais de microdureza.

Nosso objetivo foi avaliar a microdureza Knoop da resina composta fotopolimerizável a uma profundidade de $3 \mathrm{~mm}$ com corpos-de-prova confeccionados em duas diferentes matrizes de propileno, uma branca e outra preta.

\section{MATERIAL E MÉTODO}

Foram confeccionados 24 corpos-de-prova resultantes dos seis corpos-de-prova dos quatro grupos, com três medidas tomadas em cada um, totalizando 72 medidas.

Utilizamos duas matrizes desmontáveis: uma branca e outra preta constituídas de duas partes que justapostas formavam uma cavidade de formato cônico, com altura de $3 \mathrm{~mm}$, cujo diâmetro menor era de $3 \mathrm{~mm}$ e o maior de $4 \mathrm{~mm}$.

Os corpos-de-prova foram confeccionados com a resina composta $Z 100(3 \mathrm{M})$ na cor A2.

A matriz era montada e fixada numa mesa metálica para a confecção dos corpos-de-prova, onde o fundo da cavidade ficava apoiado numa lamínula de vidro.

A resina era inserida e polimerizada em incremento único de $3 \mathrm{~mm}$ e em três incrementos de $1 \mathrm{~mm}$. Para os grupos de incremento único, ela era inserida de uma só vez no interior da cavidade, preenchendo-a totalmente com uma espátula metálica e polimerizada com o aparelho fotopolimerizador XL1500 (3M) com intensidade de luz de $400 \mathrm{~mW} / \mathrm{cm}^{2}$, pelo tempo de 40 segundos.

Para os grupos com a técnica incremental utili- 
TURBINO, M. L.; SANTOS, L. A.; MATSON, E. Microdureza de resina composta fotopolimerizável: a cor da matriz experimental pode alterar os resultados dos testes? Pesqui Odontol Bras, v. 14, n. 3, p. 232-236, jul./set. 2000.

zaram-se três incrementos de $1 \mathrm{~mm}$, sendo polimerizadas por 40 segundos cada incremento.

Após o término da resina composta, cada corpo-de-prova era preso à uma Morsa Universal Ajustável (SHIMADZU) de forma que a superficie mais profunda da resina ficasse exposta e paralela ao plano horizontal da base do aparelho.

Em seguida era levado ao microdurômetro HMV 2000 SHIMADZU onde para cada corpo-de-prova eram feitas três marcações na região central. As medidas de microdureza Knoop eram obtidas com carga de 25 g por 45 segundos.

\section{RESULTADOS}

Os dados originais consistiram de 72 valores de microdureza resultantes do cruzamento de duas técnicas restauradoras, seis repetições e três medidas em cada repetição $(2 \times 2 \times 6 \times 3)$. Para análise, foram feitas as médias das 3 medidas de cada corpo-de-prova, resultando então em 24 valores. Esses dados foram analisados estatisticamente por meio do software GMC.75 sendo realizada a análise de variância. Os resultados dessa análise demonstraram que houve diferença estatisticamente significante $(\mathrm{p}<0,01)$ entre as duas cores $(F=40,42)$, sendo que a microdureza foi menor com a matriz preta $(\mathrm{m}=39,54)$ que com a branca $(\mathrm{m}=52,70)$. Entre as técnicas de inserção e polimerização, a técnica do incremento único $(\mathrm{m}=35,03)$ induziu a menores graus de polimerização $(\mathrm{p}<0,01)$ que a técnica incremental $(\mathrm{m}=57,20)$ em ambas as matrizes $(\mathrm{F}=114,76)$. Esses resultados podem ser visualizados pelas médias utilizadas no gráfico da interação técnica versus cor da matriz (Gráfico 1), onde vê-se claramente os menores valores obtidos com a matriz preta. A técnica do incremento único induziu a menores valores de dureza.

\section{DISCUSSÃO}

O grau de polimerização das resinas compostas tem sido avaliado em testes in vitro pela sua microdureza.

Como base para a confecção dos corpos-de-prova, além das cavidades realizadas em dentes, são utilizadas também matrizes, que conforme foi visto na revisão da literatura não são padronizadas quanto ao material, forma, tamanho e cor.

Devido à direta correlação entre intensidade de luz com a dureza do material $1^{4,8,13,17}$ e a profundidade de polimerização ${ }^{2,4,5,8,10,12,16,17,19}$, resultados de testes que envolvam esses fatores de variação podem ter seus valores deformados conforme o tipo de matriz experimental utilizada, tornando dificil a comparação de resultados obtidos por diferentes pesquisas.

$\mathrm{Na}$ tentativa de sugerir alguma forma de padronização desses testes, avaliamos a microdureza das resinas compostas fotopolimerizáveis com os corpos-de-prova confeccionados com matriz branca e preta. Nossos resultados comprovaram que os valores obtidos foram diferentes com as duas matrizes, sendo que quando se usou matriz branca, a dureza foi sempre maior. Supomos que a matriz preta impeça a penetração da luz pelas laterais, fazendo com que a resina receba luz apenas pela abertura da matriz. A matriz branca tendo maior grau de transmissão e reflexão da luz, induziu a maiores valores de dureza, porém corre-se o risco de não estar avaliando a real microdureza em relação à espessura da resina com determinada intensidade de luz.

Com a matriz preta, apesar dos valores diminuídos, acreditamos que a profundidade de polimerização atingida foi realmente relacionada à capacidade de penetração da luz pelo corpo da resina.

Ao avaliar-se resultados de dureza obtidos com matrizes pretas, ou que não transmitam a luz, pode-se supor que esses são os menores valores possíveis do grau de polimerização e que dependem exclusivamente das propriedades da resina e/ou do aparelho fotopolimerizador utilizados.

Quanto à utilização da técnica incremental ou do incremento único, tem-se verificado na literatura que a técnica incremental na maioria das vezes tem levado a maiores graus de polimerização e conseqüentemente maior dureza ${ }^{6,16,18}$. Em nossa pesquisa verificou-se que mesmo a técnica incre-

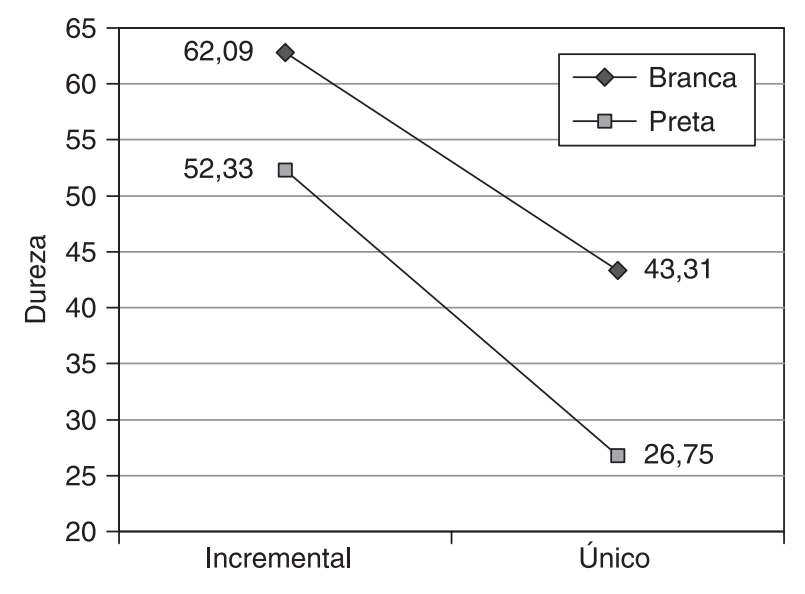

GRÁFICO 1 - Interação entre a técnica e a cor da matriz experimental. 
TURBINO, M. L.; SANTOS, L. A.; MATSON, E. Microdureza de resina composta fotopolimerizável: a cor da matriz experimental pode alterar os resultados dos testes? Pesqui Odontol Bras, v. 14, n. 3, p. 232-236, jul./set. 2000.

mental apresentou menores valores de dureza quando se usou a matriz preta em relação à branca.

Diante dessas observações, julgamos que pesquisas cujos fatores avaliados sejam relacionados à influência da luz, como profundidade de polimerização, técnica de inserção, devem criteriosamente selecionar matrizes que impeçam penetração incontrolável da luz, porém quando não houver essa preocupação, o uso de matrizes claras ou brancas não está contra-indicado.

\section{CONCLUSÕES}

- A cor da matriz experimental utilizada como base para a confecção de corpos-de-prova exerceu influência nos valores de microdureza obtidos.

- Utilizando matriz preta, os valores de dureza foram menores que os obtidos com matriz branca.

- A utilização da técnica incremental promoveu maior microdureza da resina que a técnica única para as duas cores de matriz.

TURBINO, M. L.; SANTOS, L. A.; MATSON, E. Microhardness of photopolymerized composite resin: can the color of the experimental matrix change the results of the tests? Pesqui Odontol Bras, v. 14, n. 3, p. 232-236, jul./set. 2000.

The polymerizing degree of composite resins, mainly evaluated through their microhardness, has been studied in relation to many factors. Some in vitro researches use matrices as basis for manufacturing test specimens instead of, natural teeth. For photopolymerized resins, the light intensity that reaches the material directly influences its polymerization degree. The color, as well as the transparency of the resins, should not induce differences on the final microhardness results. With this awareness in mind, this work had the aim of evaluating the effect of two polypropylene resins used in tests of composite resins. One of them was white and the other was black. The resin was applied and polymerized in a single portion of $3 \mathrm{~mm}$, and in 3 increments of $1 \mathrm{~mm}$ each, and the hardness index was measured at $3 \mathrm{~mm}$ of depth. After analyzing the results, a great difference was noticed between both colors, being the microhardness smaller in the black one. The single portion polymerization and applying techniques resulted in a smaller polymerization degree for both matrices $(\mathrm{p}<0.01)$. We could notice that, when using black matrices for manufacturing test specimens of photopolymerized composite resin, the values obtained, mainly in relation to the depth of polymerization, showed the smallest possible degree of polymerization, and they also showed that it depends only on the resin properties.

UNITERMS: Composite resins.

\section{REFERÊNCIAS BIBLIOGRÁFICAS}

1. ANDRADE, M. F.; MORONI, J. R.; CANDIDO, M. S. E. et al. Efeito da aplicação de flúor sobre a dureza superficial dos cimentos de ionômero de vidro. Rev Assoc Paul Cir Dent, v. 50, n. 2, p. 193-196, 1996.

2. ATMADJA, G.; BRYANT, R. W. Some factors influencing the depth of cure of visible light-activated composite resin. Aust Dent J, v. 35, p. 213, 1990.

3. CONCEIÇÃO, E. N.; MANFREDI, D. A. B.; PACHECO, J. F. $\mathrm{M}$. Resistência de união à dentina humana in vivo e in vitro de um sistema adesivo. In: REUNIÃO CIENTÍFICA DA SOCIEDADE BRASILEIRA DE PESQUISA ODONTOLÓGICA, 12. Águas de São Pedro, set. 1995. Anais. São Paulo : SBPqO, 1995, p. 44 [Resumo n. 87].

4. CORADAZZI, J. L.; FRANCISCHOM, C. E.; FRANCO, E. B. Avaliação de um aparelho com lâmpada halógena para polimento de RC. Rev Paul Odontol, v. 8, n. 4, p. 50-54, 1986.

5. CORRER SOBRINHO, L.; GOES, M. F.; CONSANI, S. Correlação intensidade de luz-tempo de exposição sobre a polimerização do compósito odontológico. In: REUNIÃO CIENTÍFICA DA SOCIEDADE BRASILEIRA DE PESQUISA ODONTOLÓGICA, 12. Águas de São Pedro, set. 1995. Anais. São Paulo : SBPqO, 1995, p. 60 [Resumo n. 119].

6. DE GEE, A. J.; DANDSON, A.; SMITH, A. A. Modified dilatatomer of continuous recording of volumetric polymeri- zation shrinkage of composite restorative materials. $\mathbf{J}$ Dent, v. 9, p. 36-42, 1981.

7. DREXLER, P.; GARCIA-GODOI, F.; BOJ Jr. Surface hardness of a resin composite cured with a transparent cone. Pediatr Dent, v. 19, n. 6, p. 419-420, 1997.

8. GALAN Jr., J.; BUSATO, A. L. S. Resina Composta ativada por luz visivel. R G O, v. 32, n. 4, p. 321-324, 1984.

9. LI, Y.; SWARTZ, M. L.; PHILLIPS, R. W. Effect of filler content and size on properties of composites. J Dent Res, v. 64, n. 12, p. 1396-1402, 1985.

10. MARAIS, J. T.; DANNHEIMER, M. F.; GERMISHUYS, P. J. et al. Depth of cure of light-cured composite resin with light-curing of different intensity. J Dent Assoc S Afr, v. 52, n. 6, p. 403-407, 1997.

11. MIYAZAKI, M.; MOORE, B. K.; ONOSE, H. Effect of surface coatings on flexural properties of glase ionomer. Eur $\mathbf{J}$ Oral Sci, v. 104, n. 5-6, p. 600-604, 1996.

12. PEREIRA, S. K.; PORTO, C. L. A.; MANDARINO, F. et al. Intensidade de luz e profundidade de polimerização de aparelhos fotopolimerizadores. Rev Assoc Paul Cir Dent, v. 51, n. 3, p. 257-260, 1997.

13. PILO, R.; BROSH, T.; LUGASSY, H. et al. The effect of irradiation time on the shear strength of composites. Dent Mater, v. 10, n. 6, p. 338-342, 1994.

14. QUINTELLA, L. P. A. S.; KOMATSU, J. "Inlay" de RC confeccionada em preparo cavitário isolado com água. Rev Assoc Paul Cir Dent, v. 50, n. 5, p. 403-406, 1996. 
TURBINO, M. L.; SANTOS, L. A.; MATSON, E. Microdureza de resina composta fotopolimerizável: a cor da matriz experimental pode alterar os resultados dos testes? Pesqui Odontol Bras, v. 14, n. 3, p. 232-236, jul./set. 2000.

15. RETIEF, D. H.; WENDT, S. L.; BRADLY, E. L. Effect of adhesive thickness on the shear bond strength of Scothbond 2/Silux to dentin. Am J Dent, v. 2, n. 6, p. 341-344, 1989.

16. SANTOS, L. A.; TURBINO, M. L.; YOUSSEF, M. N. et al. Microdureza de resina composta: efeito de aparelhos e tempos de polimerização em diferentes profundidades. Entregue para publicação da Pesq Odont Bras, v. 14, n. 1, p. 5-11, jan./mar. 2000.

17. SWIFT, E. J. Jr.; PAWLUS, N. A.; VARGAS, M. A. et al.
Depth of cure of resin-modified glass ionomer. Dent Mater, v. 11, n. 3, p. 196-200, 1995.

18. TURBINO, M. L. Contribuição ao estudo da microdureza Knoop de resina composta na região próxima à área de união à dentina. 1997. Tese (Doutorado) Faculdade de Odontologia, Universidade de São Paulo.

19. VINHA, D.; COÊLHO, M. T.; MAIA CAMPOS, G. Eficácia de alguns aparelhos geradores de luz visivel na polimerização da resina composta. Rev Bras Odontol, v. 47, n. 4, p. 10-14, 1990.

Recebido para publicação em 13/11/99

Enviado para reformulação em 21/02/00

Aceito para publicação em 03/04/00

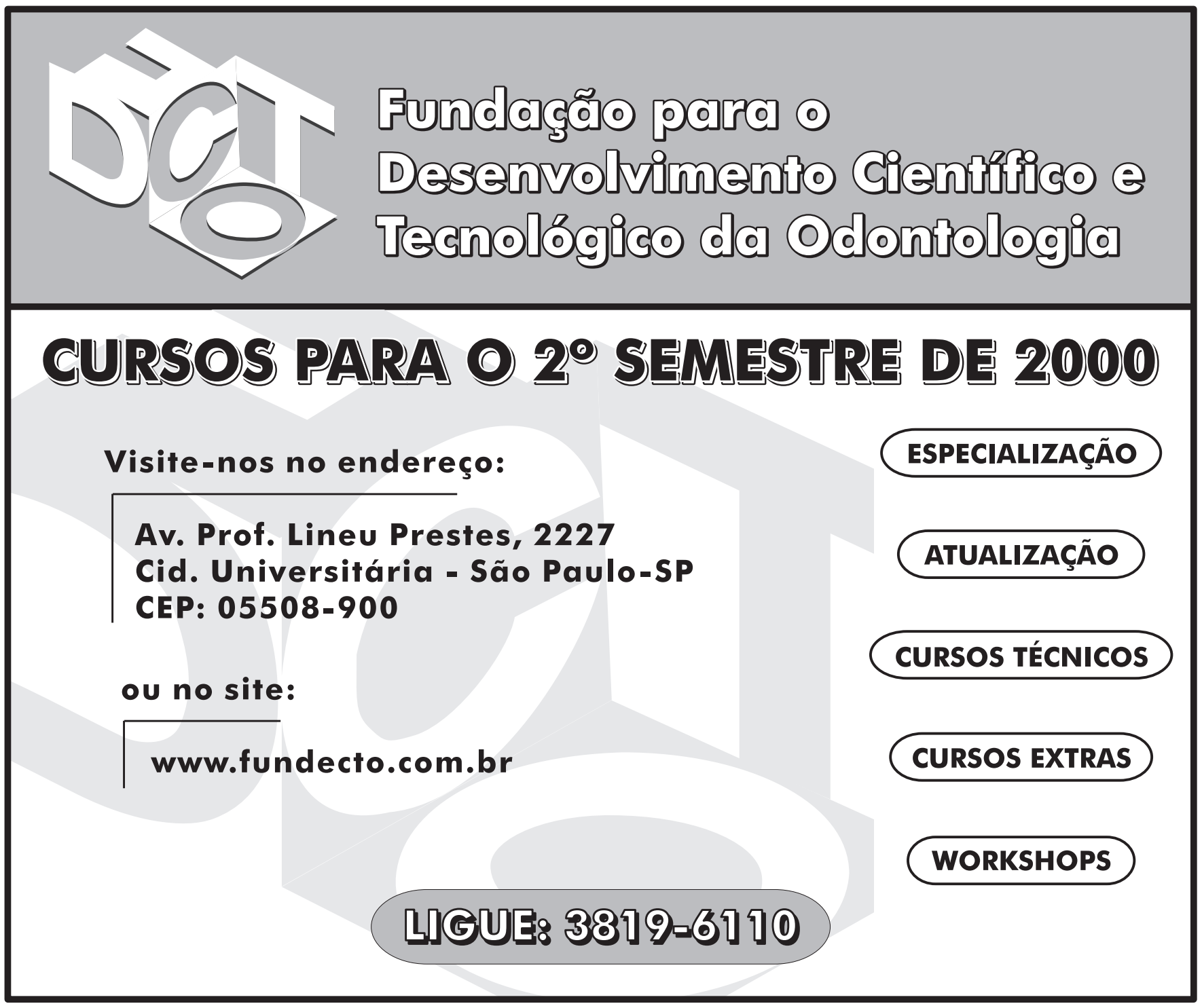

